\title{
Scale-dependent orientation in movement paths: a case study of an African viper
}

Bryan Maritz and Graham J. Alexander

\begin{abstract}
Decisions relating to the orientation of movement by animals and how this translates into movement patterns can occur at multiple spatial scales simultaneously, but this interaction is poorly understood for many groups of animals. Using the tracks left by moving snakes in their sandy habitat, we studied the movement paths of the African snake Bitis schneideri (Namaqua dwarf adder) for evidence of broad-scale directional persistence and short-range avoidance of exposure. Although snakes clearly displayed directional persistence, they preferentially moved to nearby shrubs, thereby minimizing exposure to solar and thermal radiation and/or predation. Thus, snakes made decisions relating to orientation at a minimum of two scales, the interaction of which resulted in snakes moving $\approx 17 \%$ (mean straightness index $=0.85$ ) further than the simple broad-scale straight-line distance. We assert that the actual path chosen by moving snakes represents a trade-off of various costs and risks that include risk of predation, exposure to the elements, time and energy expenditure. Our study highlights the need for cognizance of the possibility of the scale dependence of orientation and movement in studies of snake movement, and adds to a growing literature demonstrating previously unrecognized behavioural complexity in snakes.
\end{abstract}

\section{Introduction}

The way an animal moves through its environment (hereafter: movement) is a fundamental component of its life history that has broad implications for its nutritional state, mating success and risk of predation (Nathan et al. 2008). As a result, animal movement has been studied for a long time culminating in the formalization of movement ecology as an important discipline (Nathan 2008). Yet despite the growth of movement ecology as a discipline and the remarkable concomitant advances in biotelemetry (Cooke et al. 2004; Benhamou 2014), our understanding of movement for certain groups of organisms, and movement at certain spatial scales, remains superficial (Holyoak et al. 2008) and limits our ability to generalize across spatial scales or understand the evolution of such behaviour. 
Studies of fine-scale movement of vertebrates, particularly mammals and birds, have been made possible through the use of GPS telemetry (Cooke et al. 2004). For such organisms that typically range over large spatial scales (10 3 to $10^{5} \mathrm{~m}$ ), remote GPS telemetry provides appropriate data (Hulbert \& French 2001; Ryan et al. 2004) that can facilitate detailed path analysis and has driven much of our understanding of fine-scale movement by vertebrates. However, for organisms that typically move over smaller spatial scales $\left(10^{1}\right.$ to $\left.10^{2} \mathrm{~m}\right)$, GPS-derived data do not provide sufficient spatial resolution to assess movement characteristics as the spatial error associated with such data (typically $\approx 5 \mathrm{~m}$ but sometimes more; Hulbert \& French 2001) obscures short-distance movements that are likely to be common for these types of organisms. Moreover, manually mapping fine-scale movement at spatial scales appropriate to many species is logistically difficult, especially in heterogeneous environments.

In general, snakes (Reptilia: Squamata: Serpentes) are challenging to study in the wild (Seigel 1993), and our current understanding of snake movement patterns is largely due to advances in radio-telemetry techniques (Reinert 1992; Greene 1997). While these approaches have provided remarkable insights into snake behavioural and evolutionary ecology (Reinert 1992; Greene et al. 2002; Schuett et al. 2013; Pittman et al. 2014), studies of movement patterns tend to be at relatively large spatial scales $\left(10^{2}-10^{3} \mathrm{~m}\right)$ that emphasize differential habitat use, matesearching patterns, and migration between seasonal foraging-, mating- and/or overwintering-grounds (Reinert 1992; Glaudas \& Rodriguez-Robles 2011; Wastell \& Mackessy 2011; Martino et al. 2012; Miller et al. 2012; Pittman et al. 2014). Conversely, very little is known about how snakes move at fine spatial scales (i.e. at the level of actual movement paths) with most literature on path choice focussing on arboreal environments (e.g. Mansfield \& Jayne 2011; Byrnes \& Jayne 2012) or movement in relation to den sites (Landreth 1973). Additionally, little is known regarding how fine-scale movement paths reflect the various selective pressures that might be operating on individuals at different spatial scales.

Combined, the lack of information regarding fine-scale movement of snakes as a group, and small vertebrates in general, results in a significant knowledge gap in our understanding of how animals move within their environments. Here, we take advantage of several factors particular to our study species and the environment in which it occurs, which allow us to quantify fine-scale characteristics of movement paths of a small viperid snake (Namaqua dwarf adder; Bitis schneideri) by quantifying the tracks left behind in the sand by moving individuals. Namaqua dwarf adders inhabit a sandy environment punctuated with isolated shrubs (Fig. 1). Our extensive field observations suggested that snakes move directly between individual shrubs. We thus measured characteristics of movement paths of individual snakes to infer whether snakes (1) exhibited evidence for directional persistence while passing through shrubs; and (2) minimized exposure by choosing 
to move to nearby rather than distant shrubs. Adopting a 292 hypothesis contingency design (Fig. 1), we used these two lines of evidence to distinguish between four a priori hypotheses regarding fine-scale movement patterns random movement; exposure limitation (indicative of extreme exposure avoidance); direct long-range movement (indicative of long-range directional persistence); or scale-variant optimality (indicative of a trade-off between selective pressures at multiple spatial scales).

\begin{tabular}{|c|c|c|c|}
\hline & \multicolumn{2}{|c|}{ Exposure } \\
\hline & & Random & Non-random \\
\hline \multirow{2}{*}{ 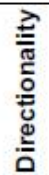 } & Random & a) Randommovement & b) Exposure limitation \\
\hline & Non-random & $\begin{array}{l}\text { c) Direct long-range } \\
\text { movement }\end{array}$ & d) Scale-variant trade-off \\
\hline
\end{tabular}

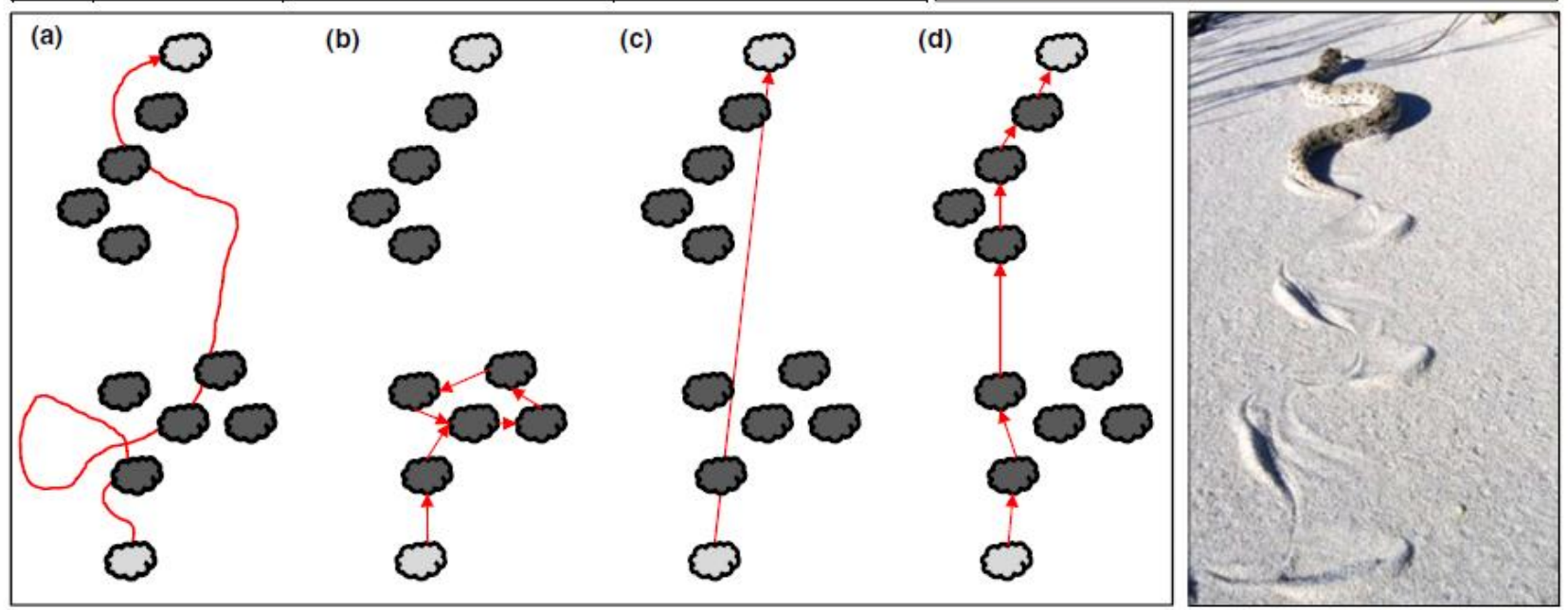

Fig. 1: (Clockwise from top left) Two-by-two contingency table explaining the four alternative hypotheses tested; typical habitat for $B$. schneideri along the South African west coast showing a matrix of shrubs and exposed sand; an adult Namaqua dwarf adder (Bitis schneideri) moves between shrubs in situ on our study site, leaving the distinctive track in the sand that we used to quantify movement metrics in this study; graphical demonstration of hypothetical paths of snakes behaving in accordance with each of the four alternative hypotheses (a-d).

\section{Methods}

\section{Study Animal}

Namaqua dwarf adders (Bitis schneideri) are diminutive, arid-adapted vipers that occur along the southern African west coast (Maritz \& Alexander 2011). They are generalist predators that ambush a range of prey species year-round from positions of concealment while buried beneath the sand with little more than their eyes exposed (Maritz \& Alexander 2014). Individuals move from one lie-up position to another with relatively short displacements (x $6.5 \mathrm{~m}$; range: $1-54 \mathrm{~m}$; N = 192), although animals travel further ( $\mathrm{x} 45.0 \mathrm{~m}$; range: $1-277 \mathrm{~m} ; \mathrm{N}=166$ ) during spring (based on raw data used in Maritz \& Alexander 2012a). Namaqua dwarf adders occupy relatively small home ranges (typically 0.1-1 ha; Maritz \& Alexander $2012 b$ ) that exhibit both sex-based and seasonal variation in size. The limited movement displayed by the snakes is likely to be at least partly induced by risk of 
predation as populations experience high annual mortality rates $(\mathbb{C}: \approx 50 \%$; range: 44-61\%; Maritz \& Alexander 2012b) and their predators are diverse and abundant (Maritz \& Scott 2010). Prolonged exposure also has thermoregulatory consequences, especially in arid environments where solar radiation is intense and surface temperatures can be extreme.

\section{Study Site}

All data were collected during summer (November to January) along coastal dune fields on the farm Noup, Northern Cape Province, South Africa $\left(30^{\circ} \mathrm{O}^{\circ} \mathrm{S}\right.$, $17^{\circ} 12^{\mathrm{O}} \mathrm{E}$ ). The region receives $50-150 \mathrm{~mm}$ rainfall per annum, and coastal fog is frequent (Cowling et al. 1999). More than 60\% of annual rainfall occurs during winter (Desmet 2007). The substrate primarily comprises recent calcareous aeolian sands that form semi-vegetated longitudinal dunes (Desmet 1996). Vegetation is succulent or sclerophyllous as is typical for Sandveld habitats along the coast (Mucina \& Rutherford 2006). Abundant plants that significantly contribute to vegetation structure include Lycium cinereum, Zygophylum morgsana, Lebeckia sericea and Ruschia robusta (Maritz 2011). In general, these shrubs occur as patches of dense vegetation in a matrix of exposed sand (Fig. 1), and are generally of approximately equal height $\left(\approx 0.5^{-1} \mathrm{~m}\right)$ due to strong prevailing winds.

\section{Path Identification and Characterization}

To record the paths taken by free-ranging snakes, we searched our study site for the characteristic tracks left by moving Namaqua dwarf adders (Fig. 1). When fresh, these tracks are unambiguous, with no other syntopic snakes leaving similar tracks. By characterizing movement metrics from tracks, we were able to infer exact movement paths without our presence disturbing the study animals while they were moving.

\section{Directionality}

To assess directional persistence, we measured the angular deviation of 102 paths through individual shrubs (Fig. 2). We excluded tracks that included lie-up positions within the shrub to minimize the potential effects of changing motivation during different bouts of movement. We tested whether angular deviations were non-randomly clustered within the available 180 degrees opposite to the point of entry into a shrub (suggesting directional persistence and navigational capacity) using Kuiper's $\mathrm{V}$ test implemented in the package 'circular' (Agostinelli \& Lund 2013) in R (R Core Team 2013).

\section{Exposure}

We chose a segment of snake track for each of 96 individuals, and using a tape measure, measured the linear length of the path taken by the snake from the point of departure of the departure shrub, to the point of entry of the destination shrub (TS; Fig. 2). Movement between shrubs was nearly always linear - although we 
did not quantify the frequency of nonlinear tracks, we estimate this frequency as less than $1 \%$ of all observed tracks during the study. Additionally, we measured the straight-line distance from the point of departure of the departure shrub along nine regularly spaced linear paths (every $22.5^{\circ}$ ), to the point of encounter with another shrub $\left(\mathrm{H}_{1}-\mathrm{H}_{9}\right.$; Fig. 2) providing us with the null distribution of available track segment lengths to which we could compare the length of the snakes chosen segment. Next, we ranked each of the nine potential paths according to their length, and calculated the mean frequency with which snakes chose each of the ranks. Using a chi-square analysis, we assessed whether snakes chose nearby shrubs more frequently than expected by chance by comparing the observed frequency with which snakes chose paths of particular ranks against a null hypothesis of all ranks being used equally.
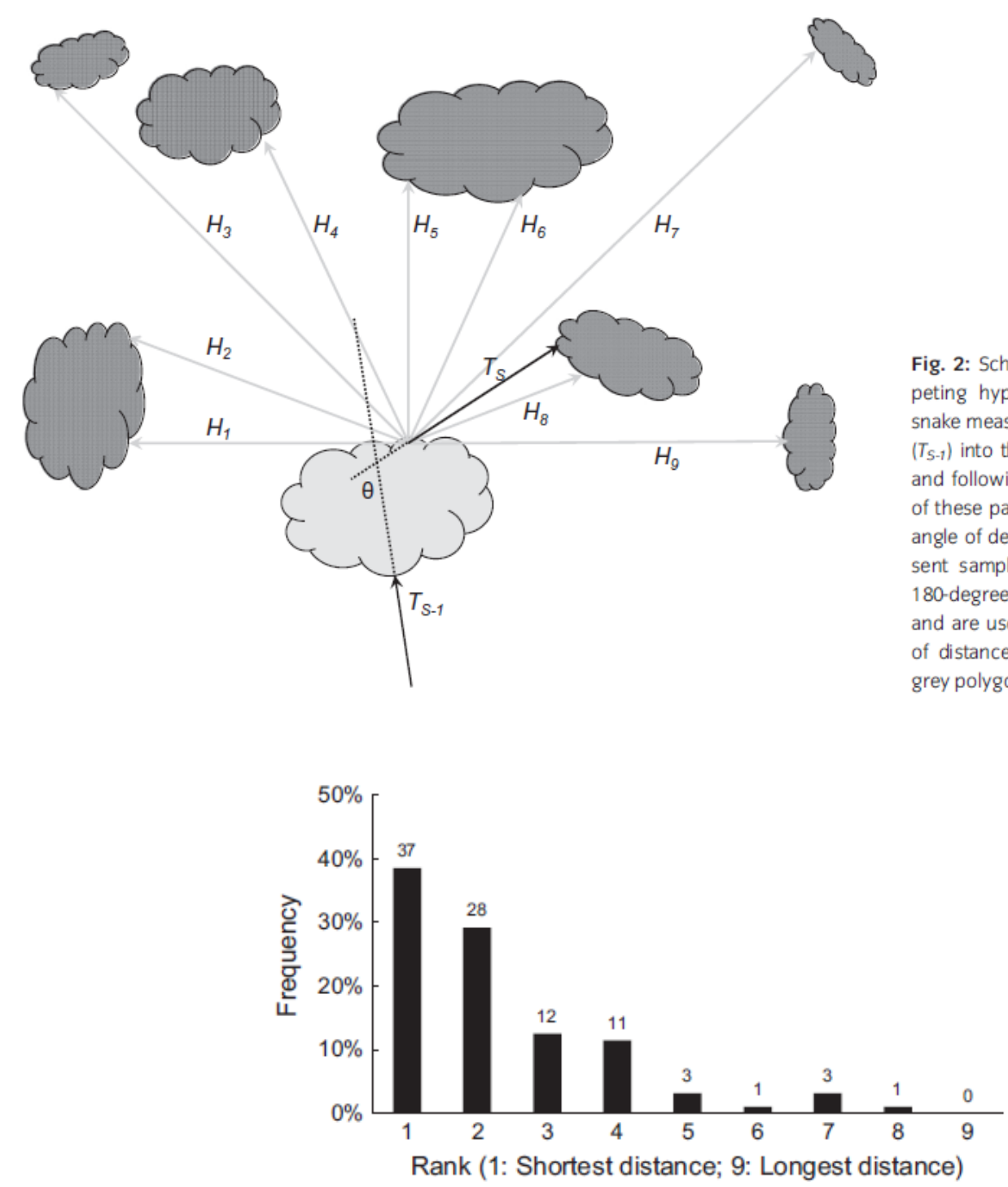

Fig. 3: Frequency distribution of the rank of track segment length $\left(T_{S}\right)$ relative to the nine hypothetical track segment lengths $\left(H_{1}-H_{9}\right)$ measured for each track studied. Numbers above bars represent number of observations for each rank.
Fig. 2: Schematic of metrics used to test competing hypotheses. The actual path of the snake measured (solid black lines) before entry $\left(T_{S-1}\right)$ into the initial shrub (light grey polygon) and following departure $\left(T_{S}\right)$. The intersection of these paths (dashed lines) used to calculate angle of deviation $(\theta) . H_{1}-H_{9}$ (grey lines) represent sample paths spaced every $22.5^{\circ}$ in a 180-degree arc around the point of departure and are used to calculate the null distribution of distances to surrounding shrubs ( $T_{N}$; dark grey polygons) available to the snake. 


\section{Straightness Index}

We measured the straightness index (Batschelet 1981) for a sample of 32 (13 adult females; 13 adult males; 6 juveniles) complete snake tracks (defined as tracks for which the entire path between the starting lie-up and the responsible animal was measurable) collected between October and March (austral spring and summer). These tracks ranged from $1.9 \mathrm{~m}$ to $48.0 \mathrm{~m}(\mathrm{x} \pm \mathrm{SD}: 11.3 \pm 9.3 \mathrm{~m})$. For each track, we divided the straight-line displacement between where the track was initially discovered and its end (where the snake was located) by the total distance moved (measured as the sum of each of the segments). We compared arcsinetransformed (arcsine of the square root of the value) mean straightness index values between adult males and adult females, and between adults and juveniles using single-factor ANOVA.

\section{Results}

We captured 39 individual snakes during fieldwork including 23 females (mean mass $=17.7 \pm 6.6 \mathrm{~g}$ ), 11 males (mean mass $=14.1 \pm 5.8 \mathrm{~g}$ ), and five juveniles (mean mass $=4.5 \pm 1.6 \mathrm{~g}$ ), suggesting that our samples were representative of the greater population (Maritz \& Alexander 2011). Snakes chose shorter (by rank) paths more frequently than longer paths $\left(\mathrm{v}^{2}=130.18\right.$; $\mathrm{p}<0.001$; Fig. 3). Mean ( \pm SD) track segment length $(\mathrm{TS}=1.03 \pm 0.75 \mathrm{~m})$ was significantly $\left(\mathrm{t}_{95}=-12.61 ; \mathrm{p}<\right.$ o.o01) shorter than mean null track segment length $\left(\mathrm{TN}_{\mathrm{N}}=1.95 \pm 0.93 \mathrm{~m}\right)$. In $39 \%$ ( $n=37$ ) of observations, the snake moved to the nearest shrub (Rank 1; Fig. 3), while movement to one of the nearest four shrubs (Ranks 1-4) accounted for 92\% $(\mathrm{n}=88)$ of all moves. Direction of departure from a shrub was also non-random (Kuiper's $\mathrm{V}_{101}=3.24 ; \mathrm{p}<0.01$ ) with most tracks maintaining their bearing (Fig. 4). Within our sample, $37 \%(\mathrm{n}=35)$ of tracks deviated by $5^{\circ}$ or less, and $72 \%(\mathrm{n}$ =69) deviated by $45^{\circ}$ or less. 


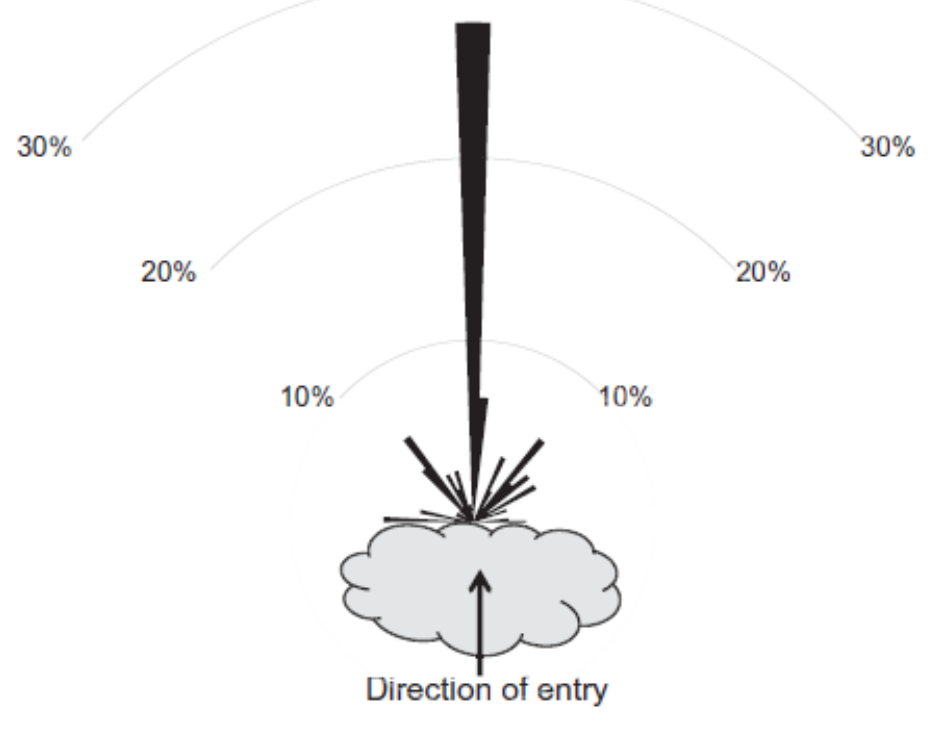

Fig. 4: Frequency distribution for angles of deviation of tracks through shrubs showing strong directional persistence. All paths standardized to a common direction of entry.

Mean straightness index for 32 complete tracks was 0.85 (range 0.56-1.00). Mean ( \pm SD) straightness index did not differ $\left(\mathrm{F}_{1,25}=0.52 ; \mathrm{p}=0.48\right)$ between adult females $(0.89 \pm 0.14)$ and adult males $(0.85 \pm 0.12)$, but was significantly lower $\left(\mathrm{F}_{1,31}=5.74 ; \mathrm{p}=0.02\right)$ in juveniles $(0.73 \pm 0.19)$.

\section{Discussion}

Our results demonstrate that Namaqua dwarf adders exhibited directional persistence when moving through the environment as well as avoidance of exposed patches. We interpret these results as evidence for individuals choosing movement paths at two spatial scales: broad-scale interpatch movement characterized by directional persistence, and fine-scale path selection biased towards nearby shrubs. Although such scale-variant decision-making is likely to be common among animals in general (Nams 2005; Nathan et al. 2008), the challenges associated with observing snakes in the wild and mapping movement patterns at a sufficiently fine spatial scale make our findings of this phenomenon in a population of snakes unique. Moreover, multiscale decision-making in a species of snake adds to a growing literature demonstrating a previously under-appreciated level of behavioural complexity among snakes (Burghardt 2013).

The ability of an animal to follow a non-random path is dependent on it being able to orient itself in space, and orientation mechanisms are also likely to be scale dependent. For example, Landreth (1973) demonstrated that directional persistence of path in the diurnal western diamondback rattlesnake (Crotalus atrox) is, at least to some extent, based on solar cues. As Namaqua dwarf adders also move almost exclusively during the day (Maritz \& Alexander 2012c), it is possible that they 
make use of this same cue for maintaining broad-scale directional persistence. However, fine-scale movements biased towards close shrubs are more likely to be dependent on the adder's ground-level view of its immediate surroundings, which is limited by its 'perceptual range' (sensu Lima \& Zollner 1996). The small body size and the restricted visual vantage of these diminutive snakes is likely to mean that their perceptual range is small (Lima \& Zollner 1996) so that even small breaks in the occurrence of shrubs could restrict movement patterns. In this context, even roads may act as barriers to movement and gene flow as they are known to in other snakes (Shine et al. 2004; Andrews \& Gibbons 2005; Andrews et al. 2008; Clark et al. 2010).

Scale-dependent optimality in movement paths results in important trade-offs for moving organisms, especially when the associated costs and risks are significant (Schooley \& Wiens 2003). Although it is likely, it is not clear whether the costs associated with broad- and fine-scale decisions in our study system represent a significant behavioural trade-off in Namaqua dwarf adders. While the cost of extended exposure at the fine spatial scale is unambiguously important to the snakes (impact on mortality), the cost of increased travelling distance, time or energy remains speculative. Our measures of mean straightness index suggest that fine-scale modification of movement reduces exposure but increases the distance by approximately $17 \%$ for a given journey. We presume that time spent moving and energy expenditure would be similarly increased. However, the hypothesis that this increased energy expenditure influences organismal fitness (and thus presents a meaningful trade-off) remains to be tested.

If meandering results in higher time and energy costs for moving from one location to another, snakes should meander the minimum needed to reduce exposure, although actively foraging and mate-searching snakes may follow a more tortuous path (Mueller \& Fagan 2008; non-oriented mechanisms) if resources are patchy. Our measures of meandering in Namaqua dwarf adders are low (Secor 1994 reports meander ratio, the inverse of the straightness index, of >2:1 for Crotalus cerastes, corresponding to a straightness index of $>0.5$ ), and are in keeping with those expected for an ambush forager moving from one location to another. We recorded significantly more meandering (lower straightness index) in juveniles, possibly due to an increased need for juveniles to avoid exposure to thermal radiation and possibly accounting for the lower detection probabilities recorded by Maritz \& Alexander (2012b). Although Secor (1994) found lower meander ratios for neonate C. cerastes, he did not find differences between juveniles and adults. Clearly, several factors could impact the trade-off of costs and benefits of an increased meander ratio, but this has not been studied in snakes.

We interpret short-range selection of cover as being driven by at least two nonexclusive mechanisms. Unpublished thermal data from our study site show that daytime surface temperature in exposed habitat patches frequently exceed $50^{\circ} \mathrm{C}$ 
but is significantly lower in shrubs. During midday, patches of exposed habitat are thus inhospitable or even lethal to snakes, especially as the small body size of Namaqua dwarf adders is likely to result in rapid heating in such conditions. Despite moving only during the day, individuals avoid moving during the hottest periods (Maritz \& Alexander 2012c), highlighting the importance of the thermal environment in governing movement patterns. Unfortunately, the use of tacks in the current study did not allow us to ascertain what time of the day or the temperature when tracks were made, limiting our ability to test the thermal inhospitablity hypothesis.

Several observations suggest predator avoidance as a primary motivation for shortrange decisions to minimize exposure. Firstly, mark-recapture analysis reveals that Namaqua dwarf adder populations experience very high annual mortality rates (Maritz \& Alexander 2012b) resulting from diverse and abundant predators with which they coexist (Maritz \& Scott 2010; Maritz 2011). Secondly, Namaqua dwarf adders show strong background colour-matching and disruptive colouration, likely arising as camouflage against predation (B. Maritz, pers. obs). Finally, examination of lie-up sites reveals that snakes generally lie-up adjacent to, even small objects such as sticks or small dislodged branches that appear to act as disruptive camouflage against visual predators.

Although our approach provides insight into the movement patterns of a cryptic organism, it is relatively simplistic and thus has several limitations. For example, data on the time of the day that movements took place could help differentiate between thermally induced and predation-induced motivations for avoiding exposed areas, while information on sex and age-class could shed light on motivations for movement across broad spatial scales. Given the relative structural homogeneity of the habitat, it seems unlikely that interpatch movement is associated with broad-scale structural components across the landscape. Rather, we speculate that such movement is more likely to be associated with resource detection, either as mates or foraging sites.

This study is the first to directly demonstrate that snakes make decisions on directionality of movement at multiple spatial scales. Our results indicate that studies of snake movement should take cognizance of the possibility of scale dependence in mechanisms of orientation and movement. Detecting the relevant scales at which decisions about movement are made is crucial to understanding many ecological processes (Benhamou 2014). Our findings suggest that Namaqua dwarf adders make scale-specific decisions that may drive fine-scale movement patterns, but maintain broad-scale directionality. In doing so, we add to a growing literature demonstrating previously unrecognized behavioural complexity in snakes. Moreover, our findings provide insight into movement patterns for a cryptic and difficult to study group of organisms that often move at relatively small spatial scales that have rarely been examined by movement ecologists. 


\section{Acknowledgements}

We thank E. Oppenheimer and Son, the Rufford Small Grants Foundation and the South African National Biodiversity Institute for financial support. All work was cleared under Northern Cape Province Department of Tourism, Environment and Conservation (0914/07 and FAUNA 698/2009) and University of the Witwatersrand Animal Ethics Screening Committee (2007/68/1 and 2007/69/3) permits. Michael Pierce, Alexander Haw and Bruce Wessels helped gather field data. 


\section{Literature cited}

Agostinelli, C. \& Lund, U. 2013: R package 'circular': Circular Statistics (version 0.47).

Andrews, K. M. \& Gibbons, J. W. 2005: How do highways influence snake movement? Behavioral responses to roads and vehicles. Copeia 2005, 772782.

Andrews, K. M., Gibbons, J. W. \& Jochimsen, D. M. 2008: Ecological effects of roads on amphibians and reptiles: a literature review. In: 'Urban Herpetology' Herpetological Conservation (Mitchell, J. C., Jung Brown, R. E. \& Bartholomew, B., eds.) SSAR Books, Salt Lake City, pp 121-143.

Batschelet, E. 1981: Circular Statistics in Biology. Academic Press, London.

Benhamou, S. 2014: Of scales and stationarity in animal movements. Ecol. Lett. $17,261-272$.

Burghardt, G. M. 2013: Environmental enrichment and cognitive complexity in reptiles and amphibians: concepts, review, and implications for captive populations. Appl. Anim. Behav. Sci. 147, 286-298.

Byrnes, G. \& Jayne, B. C. 2012: The effects of three-dimensional gap orientation on bridging performance and behavior of brown tree snakes (Boiga irregularis). J. Exp. Biol. 215, 2611-2620.

Clark, R. W., Brown, W. S., Stechert, R. \& Zamudio, K. R. 2010: Roads, interrupted dispersal, and genetic diversity in timber rattlesnakes. Conserv. Biol. 24, 1059-1069.

Cooke, S. J., Hinch, S. G., Wikelski, M., Andrews, R. D., Kuchel, L. J., Wolcott, T. G. \& Butler, P. J. 2004: Biotelemetry: a mechanistic approach to ecology. Trend. Ecol. Evol. 19, 334-343.

Cowling, R. M., Esler, J. \& Rundel, P. W. 1999: Namaqualand, South Africa - an overview of a unique winter-rainfall desert ecosystem. Plant Ecol. 142, 3-21.

Desmet, P. G. 1996: Vegetation and restoration potential of the arid coastal belt between Port Nolloth and Alexander Bay, Namaqualand, South Africa. MSc Thesis, University of Cape Town, Cape Town.

Desmet, P. G. 2007: Namaqualand - A brief overview of the physical and floristic environment. J. Arid Environ. 70, 570-587.

Glaudas, X. \& Rodriguez-Robles, J. A. 2011: Vagabond males and sedentary females: spatial ecology and mating system of the speckled rattlesnake (Crotalus mitchellii). Biol. J. Linn. Soc. 103, 681-695.

Greene, H. W. 1997: Snakes: The Evolution of Mystery in Nature. University of California Press, Berkeley.

Greene, H. W., May, P. G., Hardy, D. L. Sr, Sciturro, J. M. \& Farrell, T. M. 2002: Parental behavior by vipers. In: Biology of the Vipers. (Schuett, G. W., Hoggren, M., Douglas, M. E., Greene, H. W., eds). Eagle Mountain Publishing, Utah, pp. 179-205.

Holyoak, M., Casagrandi, R., Nathan, R., Revilla, E. \& Spiegel, O. 20o8: Trends and missing parts in the study of movement ecology. Proc. Natl Acad. Sci. USA 105, 19060-19065. 
Hulbert, I. A. \& French, J. 2001: The accuracy of GPS for wildlife telemetry and habitat mapping. J. Appl. Ecol. 38, 869-878.

Landreth, H. F. 1973: Orientation and behavior of the rattlesnake, Crotalus atrox. Copeia 1973, 26-31.

Lima, S. L. \& Zollner, P. A. 1996: Towards a behavioral ecology of ecological landscapes. Trend. Ecol. Evol. 11, 131-135.

Mansfield, R. H. \& Jayne, B. C. 2011: Arboreal habitat structure affects route choice by rat snakes. J. Comp. Physiol. A. 197, 119-129.

Maritz, B. 2011: Ecology of the Namaqua Dwarf Adder, Bitis schneideri. PhD Thesis, University of the Witwatersrand, Johannesburg.

Maritz, B. \& Alexander, G. J. 2011: Morphology, sexual dimorphism, and growth in the smallest viperid, Bitis schneideri (Reptilia: Squamata: Viperidae). J. Herpetol. 45, 457-462.

Maritz, B. \& Alexander, G. J. 2012a: Dwarfs on the move: spatial ecology of the world's smallest viper, Bitis schneideri. Copeia 2012, 115-120.

Maritz, B. \& Alexander, G. J. 2012b: Population density and survival estimates of the African viperid, Bitis schneideri. Herpetologica 68, 195-202.

Maritz, B. \& Alexander, G. J. 2012c: Movement patterns in the smallest viper, Bitis schneideri. Copeia 2012, 732- 737.

Maritz, B. \& Alexander, G. J. 2014: Namaqua Dwarf Adders are generalist predators. Afr. J. Herpetol. 63, $79-86$.

Maritz, B. \& Scott, S. L. 2010: Assessing the threat of avian predation on a small viperid snake. Afr. Zool. 45, 309-314.

Martino, J. A., Poulin, R. G., Parker, D. L. \& Somers, C. M. 2012: Habitat selection by grassland snakes at northern range limits: implications for conservation. J. Wildlife Manage. 76, 759-767.

Miller, G. J., Smith, L. L., Johnson, S. A. \& Franz, R. 2012: Home range size and habitat selection in the florida pine snake (Pituophis melanoleucus mugitus). Copeia 4, 706- 713 .

Mucina, L. \& Rutherford, M. C. 2006: The vegetation of South Africa, Lesotho and Swaziland. Strelitzia 19. SANBI, Pretoria, South Africa.

Mueller, T. \& Fagan, W. F. 2008: Search and navigation in dynamic environments - from individual behaviors to population distributions. Oikos 117, 654-664.

Nams, V. O. 2005: Using animal movement paths to measure response to spatial scale. Oecologia 143, 179-188.

Nathan, R. 2008: An emerging movement ecology paradigm. Proc. Natl Acad. Sci. USA 105, 19050-19051.

Nathan, R., Getz, W. M., Revilla, E., Holyoak, M., Kadmon, R., Saltz, D. \& Smouse, P. E. 2008: A movement ecology paradigm for unifying organismal movement research. Proc. Natl Acad. Sci. USA 105, 19052-19059.

Pittman, S. E., Hart, K. M., Cherkiss, M. S., Snow, R. W., Fujisaki, I., Smith, B. J., Mazzotti, F. J. \& Dorcas, M. E. 2014: Homing of invasive Burmese pythons in South Florida: evidence for map and compass senses in snakes. Biol. Lett. 10, 20140040. 
R Core Team 2013: R: A Language and Environment for Statistical Computing. R Foundation for Statistical Computing, Vienna, Austria. URL http://www.Rproject. org/.

Reinert, H. K. 1992: Radiotelemetric field studies of pitvipers: data acquisition and analysis. In: Biology of the Pitvipers (Campbell, J. A. \& Brodie, E. D. Jr, eds). Selva Press, Tyler, Texas, pp. 185-197.

Ryan, P. G., Petersen, S. L., Peters, G. \& Gremillet, D. 2004: GPS tracking a marine predator: the effects of precision, resolution and sampling rate on foraging tracks of African penguins. Mar. Biol. 145, 215-223.

Schooley, R. L. \& Wiens, J. A. 2003: Finding habitat patches and directional connectivity. Oikos 102, 559-570.

Schuett, G. W., Repp, R. A., Hoss, S. K. \& Herrmann, H.-W. 2013: Environmentally cued parturition in a desert rattlesnake, Crotalus atrox. Biol. J. Linn. Soc. 110, 866-877.

Secor, S. M. 1994: Ecological significance of movements and activity range for the sidewinder, Crotalus cerastes. Copeia 1994, 631-645.

Seigel, R. A. 1993: Summary: future research on snakes, or how to combat 'lizard envy'. In: Snakes: Ecology and Behavior. (Seigel, R. A., Collins, J. T., Novak, S. S., eds). Macmillan Publishing Company, New York, pp. 395- 402.

Shine, R., LeMaster, M., Wall, M., Langkilde, T. \& Mason, R. 2004: Why did the snake cross the road? Effects of roads on movement and location of mates by garter snakes (Thamnophis sirtalis parietalis). Ecol. Soc. 9, 9.

Wastell, A. R. \& Mackessy, S. P. 2011: Spatial ecology and factors influencing movement patterns of desert massasauga rattlesnakes (Sistrurus catenatus edwardsii) in south-eastern Colorado. Copeia 1, 29-37. 